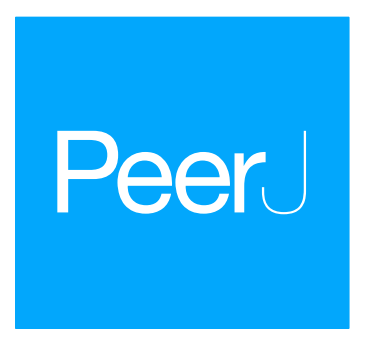

Submitted 22 March 2016

Accepted 26 July 2016

Published 25 August 2016

Corresponding author

Navjot Bhullar,

navjot.bhullar@une.edu.au

Academic editor

Nicola Reavley

Additional Information and Declarations can be found on page 9

DOI 10.7717/peerj.2373

Copyright

2016 MacDonell et al.

Distributed under

Creative Commons CC-BY 4.0

OPEN ACCESS

\section{Depression, anxiety, and stress in partners of Australian combat veterans and military personnel: a comparison with Australian population norms}

\author{
Gail V. MacDonell ${ }^{*}$ Navjot Bhullar* and Einar B. Thorsteinsson*
}

Psychology-School of Behavioural, Cognitive and Social Sciences, University of New England, Armidale, New South Wales, Australia

These authors contributed equally to this work.

\section{ABSTRACT}

Partners of Australian combat veterans are at an increased risk of experiencing mental health problems. The present study provides a comparative analysis of the mental health of partners of veterans with that of the Australian normative data. To compare different types of groups of partners, the study samples comprised: (a) partners of Australian combat veterans (Sample 1: $n=282$, age $M=60.79, S D=5.05$ ), (b) a subsample of partners of Australian combat veterans from the previous sample (Sample 2: $n=50 ; M=60.06, S D=4.80$ ), (c) partners of Special Air Services Regiment (SASR) personnel (Sample 3: $n=40$, age $M=34.39 S D=7.01$ ), and (d) partners of current serving military (non-SASR) personnel (Sample $4: n=38$, age $M=32.37, S D=6.20$ ). Respondents completed measures assessing their reported levels of depression, anxiety, and stress. Samples 1 and 2 comprised partners of Australian military veterans who reported significantly greater symptoms of depression, anxiety, and stress than the comparative population norms. The sample of SASR personnel partners (Sample 3 ) reported significantly lower levels of depression and anxiety, whereas the sample with non-SASR personnel partners (Sample 4) reported a significantly greater stress symptomatology than the comparative norms. Number of deployments was found to be associated with depression, anxiety, and stress in partners of non-SASR veterans (Sample 4). Lessons and protective factors can be learnt from groups within the current military as to what may assist partners and families to maintain a better level of psychosocial health.

Subjects Global Health, Health Policy, Nursing, Psychiatry and Psychology, Public Health Keywords Dyadic adjustment, Combat veterans, Caregiving distress, Mental health, Partners of veterans, PTSD

\section{INTRODUCTION}

Partners of military and combat veterans ${ }^{1}$ are at a risk of experiencing higher levels of mental health distress symptomatology (Alessi et al., 2001). Progress has been made in research of partners of veterans regarding their psychosocial outcomes and the major issues they face (e.g., Beckham, Lytle \& Feldman, 1996; Calhoun, Beckham \& Bosworth, 2002; MacDonell et al., 2014; Outram et al., 2009; Renshaw \& Campbell, 2011; Renshaw \& Caska,

${ }^{1}$ In this paper, we referred to all Military personnel as combat veterans. 
2012; Solomon et al., 1991; Westerink \& Giarratano, 1999). Evidence suggests that partners of veterans with Posttraumatic Stress Disorder (PTSD) showed higher levels of emotional distress than those in the non-military population (Dekel et al., 2005a), and that partners of veterans with PTSD experienced higher levels of caregiver burden and lower levels of psychological adjustment than those partners whose veteran did not have PTSD (Calhoun, Beckham \& Bosworth, 2002). The majority of this research has compared the partners of veterans who reported to have PTSD with that of partners without PTSD. However, no research to date has compared the partners of different services (e.g., regiments) personnel; therefore, the present paper examined the mental health of partners of veterans from three different military services and compared them with the Australian population norms.

There is a growing interest in understanding the relationship between veterans' deployment stressors and exposure to combat and their partners' risk for mental health and dyadic adjustment problems (Calhoun, Beckham \& Bosworth, 2002). The personal and family relationships of veterans are often marked by considerable distress and dyadic maladjustment (MacDonell et al., 2010; MacDonell et al., 2014; Outram et al., 2009). Findings from the United States, Israel, Holland, Croatia, Iran (Calhoun, Beckham \& Bosworth, 2002; Dekel et al., 2005a; Dirkzwager et al., 2005; Frančeikovišć et al., 2007; Salimi et al., 2006) and Australia (MacDonell et al., 2010; MacDonell et al., 2014; Outram et al., 2009; Westerink \& Giarratano, 1999) have suggested that partners of combat veterans have a significantly higher risk of developing psychosocial problems as a result of living with and caring for their veterans, particularly those veterans with PTSD. Moreover, it has been shown that the psychosocial functioning of partners is poorer than the general population overall (Beckham, Lytle \& Feldman, 1996; MacDonell et al., 2010; Westerink \& Giarratano, 1999). Research has found that the greater the symptoms of PTSD of the veterans, the greater the distress in their partners (Dekel, Solomon \& Bleich, 2005b; Pearlin et al., 1990). The long-term effects of PTSD have shown to be labile, but indicate that the detrimental effects are complex, deep and enduring (Solomon et al., 2014; Solomon \& Mikulincer, 2006).

Research has also shown that multiple deployments for veterans tend to lead to higher rates and severity of PTSD (Hoops, 2012; Kline et al., 2010) and that the military lifestyle can have negative outcomes on the family, particularly the spouse or partner (Burrell et al., 2006). Separation (not only from deployment), unpredictable duty hours, frequent relocations and single parenting (parenting while the veteran is away either being deployed or on training courses) are just a few of the stressors that face partners of veterans on a regular basis (Padden, Connors \& Agazio, 2011). Moreover, attempting to build a career while being a partner of a veteran is difficult, with some suggesting that existing gender inequality in the workplace gives these partners a dual disadvantage (Doherty, Patton \& Shield, 2015). There is also evidence that partners' behavioral health is becoming more problematic (Ahmadi \& Green, 2011), including increases in drug and alcohol use. Research has started to recognise that the partner of a veteran plays a crucial role in the health of the veteran and the couple's relationship (Lewis, Lamson \& Leseuer, 2012). However, there is limited research into the long-term effects on the partners and families, over the lifespan of the relationships (Link \& Palinkas, 2013), therefore, more research is needed to examine 
the possible negative feedback loop between family factors that affect the combat veterans and their families.

The present paper investigated the degree of psychological distress by examining levels of depression, anxiety and stress in partners of veterans serving in three different military services, and compares them with norms for the Australian population. We specifically examined the similarities and differences between different samples of partners of veterans: (a) partners of veterans (POV) who have left the military (Samples 1 and 2); (b) current serving Special Air Services Regiment (SASR, Sample 3); and (c) currently serving military who are partners of non-SASR veterans (Sample 4). A description of all the four samples is provided in Method section. Previous research also suggests that a greater number of deployments is associated with higher levels of PTSD symptomology in combat veterans which, in turn, may have a negative impact on their partners' mental health outcomes. Although, no official data on deployments is currently available, the anecdotal evidence suggests that SASR personnel experience more deployments than other military personnel. Therefore, we predicted that the partners of SASR veterans would report significantly greater psychological distress as compared with other samples of partners.

\section{METHOD}

\section{Participants Sample 1}

Participants were 282 female partners of Australian veterans (i.e., partners of veterans (POV)). These participants were members of the Partners of Veterans Association of Australia (PVA). Membership in this association is open to all partners of current and former partners (those who may have separated from their veteran spouse or who are widowed) who served in the theatre of war or campaign, or who are currently serving in any peacekeeping, peace-making, or operational service. Meetings in different areas are usually held on a monthly or more regular basis as a way of support and air any problems the partners may have. Participants were recruited via the PVA newsletter and they completed a self report questionnaire. Partners' ages ranged from 43 to 83 years $(M=60.79, S D=5.05)$. Their length of marriage ranged from seven to 60 years $(M=35.43, S D=9.74)$. Participants reported that their veterans had served in the Air Force (12\%), Army (82\%), and Navy (6\%). Just over half (53\%) of the veterans had been long-term service personnel, with $44 \%$ being National Conscripts, 3\% where National Conscripts who stayed on to become long term service personnel. The percentage of veterans who attended different conflict zones was 1\% in World War II, 2\% in Korea, 9\% in Malaya/Borneo, $82 \%$ in Vietnam, $0.5 \%$ each in Somalia, Sinai, Kashmir, Gulf War 1 and East Timor, whereas 1\% indicated 'other' as their first conflict. Of those who indicated that their veteran had been deployed in an active service zone more than once, $68 \%$ had been deployed twice, $9 \%$ three times, $9 \%$ four times and $4 \%$ five times.

\section{Sample 2 (as second POV sample)}

The first 50 participants who were still living with a veteran at the time of data collection were selected from Sample 1. POV female partners' ages ranged from 43 to 70 years 
$(M=60.06, S D=4.80)$. Their length of marriage ranged from seven to 47 years $(M=34.22, S D=10.41)$. Of those five indicated that their veteran had been deployed in an active service zone more than once, 20 twice, five 3-times, five 4-times, and five 5-times. Ten did not indicate their partners' number of deployments.

\section{Sample 3}

These were 40 female partners of Special Air Service Regiment (SASR) personnel. These partners were a convenience sample who completed an online survey over a period of two weeks following meetings discussing their issues. The SASR personnel partners' ages ranged from 23 to 49 years $(M=34.39, S D=7.01)$. The length of their relationship ranged from 1 to 30 years $(M=11.66, S D=7.53)$. In Sample 3, two veterans had not been deployed, two had been deployed once, one twice, six 3-times, four 4-times, six 5-times, and two 7-times, five 8-times, four 9-times, two 10-times and six had been on deployed over 12-times.

\section{Sample 4}

This group consisted of 38 female partners of current Defence personnel (non-SASR). This was a convenience sample completing an online survey over a period of two weeks following meetings discussing their issues. Defence partners' (non-SASR) ages ranged from 22 to 48 years $(M=32.37, S D=6.20)$. The length of the relationship ranged from two to 23 years $(M=9.55, S D=5.07)$. Ten defence personnel had been deployed once, seven twice, thirteen 3-times, one 5-times, one 7-times, one 8-times, and five had been deployed more than 12-times.

\section{Measures}

\section{The Depression Anxiety Stress Scales (DASS)}

The 21-item DASS (Lovibond \& Lovibond, 1995a; Lovibond \& Lovibond, 1995b) was used to assess levels of depression, anxiety, and stress. This self-report scale comprises three subscales: depression, anxiety and stress. Each subscale consists of seven items, answered on a Likert like scale from 0 ("Did not apply to me at all") to 3 ("Applied to me very much, or most of the time"). A sample item for Depression is "I couldn't seem to experience any positive feeling at all"; for Anxiety: "I was aware of dryness of my mouth", and for Stress: "I found it difficult to relax". Low scores on these subscales reflect better mental health. Antony et al. (1998) validated the scale showing that depressive patients scored highest on the depression and stress subscales, whereas panic disorder patients scored highest on the anxiety subscale. The depression subscale measures dysphoria, hopelessness, anhedonia and inertia, whereas the anxiety subscale assesses autonomic arousal, skeletal musculature effects, situational anxiety and subjective experience of anxious effect (feelings of panic and fear). The stress subscale assesses difficulty in relaxing, impatience and chronic non-specific arousal (Lovibond \& Lovibond, 1995a). The totals of each subscale were multiplied by two to reflect the scores of the DASS 42 and referenced to the normative data (Lovibond \& Lovibond, 1995a).

For depression, anxiety, and stress, Cronbach's $\alpha$ s for Sample 1 (POV) were .94, .90 and .91 , respectively; for Sample 2 (second POV sample), $\alpha$ s were $.86, .87$ and .81 , respectively; 


\begin{tabular}{|c|c|c|c|c|c|c|c|}
\hline Measure & $\operatorname{POV} M(S D)$ & Norms $M(S D)$ & $t(281)$ & $\begin{array}{l}\text { Mean } \\
\text { difference }\end{array}$ & $\begin{array}{l}\text { 95\% CI } \\
\text { (Mean difference) }\end{array}$ & Hedges' $g$ & $\begin{array}{l}\text { 95\% CI } \\
\text { (Hedges' } g \text { ) }\end{array}$ \\
\hline Depression & $16.09(12.58)$ & $6.14(6.92)$ & $13.27^{*}$ & 9.95 & $8.47,11.42$ & 0.79 & $0.62,0.96$ \\
\hline Anxiety & $12.61(11.83)$ & $4.80(4.70)$ & $11.08^{*}$ & 7.81 & $6.42,9.19$ & 0.66 & $0.49,0.83$ \\
\hline Stress & $19.69(11.71)$ & $10.29(8.16)$ & $13.47^{*}$ & 9.39 & $8.02,10.77$ & 0.80 & $0.63,0.97$ \\
\hline
\end{tabular}

Notes.

Norms based on Females' normative data sample $(N=1,870)$, Lovibond \& Lovibond (1995b). Hedges' effect size: small (0.2) medium (0.5) and large (0.8). Tests on the four groups were conducted using the Bonferroni adjustment alpha levels of .016 (.05/3).

${ }^{*} p<.001$, two-tailed.

for Sample 3 (SASR), $\alpha$ s were .78, .62, and.62, respectively, and Sample 4 (non-SASR), $\alpha$ s were $.96, .88$, and .84 , respectively.

\section{Procedure}

Participants in Sample 1 (POV) were asked to complete a self-report questionnaire that was sent out in a national newsletter to partners of veterans, as part of a larger study (MacDonell et al., 2014). Both SASR and non-SASR (Samples 3 and 4) participants were convenience samples and were asked to fill out a questionnaire online over a 2-week period. Sample 2 participants who were still living with a veteran at the time of data collection were selected from Sample 1 to create a POV sample comparative in number with Samples 3 and 4. Ethics approval was given by Human Research Ethics Committee, University of New England, and approval number HE09/151. Participants gave their consent by either completing the hard-copy of the questionnaire or clicking a 'proceed' button on the online questionnaire after reading the information sheet for the study.

\section{Statistical analysis}

All statistical analyses were run using SPSS version 22 using two datasets, one with Sample 1 (MacDonell, Bhullar \& Thorsteinsson, 2016a) and one with Samples 2, 3, 4 combined (MacDonell, Bhullar \& Thorsteinsson, 2016b). For the comparative analyses, a series of one-sample $t$-tests were conducted with Bonferroni adjustments where applicable. All missing data on study variables $(<3 \%)$ were considered missing completely at random and were replaced with values computed by the expectation maximization algorithm in SPSS (Tabachnick \& Fidell, 2001).

\section{RESULTS}

The reported levels of depression, anxiety, and stress were moderately higher, than Australian female normed values, in Sample 1 (Table 1) and Sample 2 (Table 2). Our results indicated that there were no statistical differences for depression, anxiety, and stress between Samples 1 and 2 (both POV samples).

The findings for partners of Australian SASR members (Sample 3) showed that they had lower levels of depression and anxiety than the normative data (see Table 3). Comparing normative data of depression, anxiety, and stress levels in partners of Australian current serving non-SASR members (Sample 4) showed a small significant difference for stress. 


\begin{tabular}{|c|c|c|c|c|c|c|c|}
\hline Measure & POV M(SD) & Norms $M(S D)$ & $t(49)$ & $\begin{array}{l}\text { Mean } \\
\text { difference }\end{array}$ & $\begin{array}{l}\text { 95\% CI } \\
\text { (Mean difference) }\end{array}$ & Hedges' $g$ & $\begin{array}{l}95 \% \text { CI } \\
\text { (Hedges' } g \text { ) }\end{array}$ \\
\hline Depression & $18.84(12.27)$ & $6.14(6.92)$ & 7.32 & 12.70 & $9.21,16.19$ & 0.44 & $0.27,0.60$ \\
\hline Anxiety & $12.88(11.00)$ & $4.80(4.70)$ & $5.19^{*}$ & 8.08 & $4.95,11.22$ & 0.31 & $0.41,0.48$ \\
\hline Stress & $22.68(11.58)$ & $10.29(8.16)$ & $7.56^{*}$ & 12.39 & $9.09,15.68$ & 0.45 & $0.28,0.62$ \\
\hline
\end{tabular}

Notes.

Norms based on Females normative data sample $(N=1,870)$ Lovibond \& Lovibond (1995b). Hedges' effect size: small (0.2) medium (0.5) and large (0.8). Tests on the four groups were conducted using the Bonferroni adjustment alpha levels of .016 (.05/3).

${ }^{*} p<.001$, two-tailed.

Table 3 Summary statistics for DASS for sample 3 Special Air Service Regiment (SASR; $n=40)$ and DASS normative data.

\begin{tabular}{llllllll} 
Measure & SASR $\boldsymbol{M}(\boldsymbol{S D})$ & Norms $\boldsymbol{M}(\boldsymbol{S D})$ & $\boldsymbol{t}(\mathbf{3 9})$ & $\begin{array}{l}\text { Mean } \\
\text { difference }\end{array}$ & $\begin{array}{l}\mathbf{9 5 \%} \text { CI } \\
\text { (Mean difference) }\end{array}$ & $\begin{array}{c}\text { Hedges' } \boldsymbol{g} \\
\text { 95\% CI } \\
(\text { Hedges' } \boldsymbol{g})\end{array}$ \\
\hline Depression & $4.15(4.58)$ & $6.14(6.92)$ & -2.75 & -1.99 & $-3.46,-0.52$ & -0.16 & $-0.33,0.00$ \\
Anxiety & $2.45(5.39)$ & $4.80(4.70)$ & $-2.76^{*}$ & -2.35 & $-4.07,-0.63$ & -0.16 \\
Stress & $9.65(7.62)$ & $10.29(8.16)$ & -0.53 & -0.64 & $-3.08,1.79$ & $-0.33,0.00$ \\
\hline
\end{tabular}

Notes.

Norms based on Females' normative data sample $(N=1,870)$, Lovibond \& Lovibond (1995b). Hedges' effect size: small (0.2) medium (0.5) and large (0.8). Number of participants is 40 rather than 41 as one participant did not answer the DASS questionnaire. Tests on the four groups were conducted using the Bonferroni adjustment alpha levels of $.016(.05 / 3)$.

${ }^{*} p<.012$, two-tailed.

Table 4 Summary statistics for DASS for Sample 4 of current serving non-Special Air Service Regiment (non-SASR; $n=38)$ and DASS normative data.

\begin{tabular}{llllllcc} 
Measure & $\begin{array}{l}\text { Non-SASR } \\
\boldsymbol{M}(\boldsymbol{S D})\end{array}$ & $\begin{array}{l}\text { Norms } \\
\boldsymbol{M}(\boldsymbol{S D})\end{array}$ & $\boldsymbol{t}(\mathbf{3 7})$ & $\begin{array}{l}\text { Mean } \\
\text { difference }\end{array}$ & $\begin{array}{l}\mathbf{9 5 \%} \text { CI } \\
\text { (Mean difference) }\end{array}$ & $\begin{array}{l}\text { Hedges' } \boldsymbol{g} \\
\text { 95\% CI } \\
(\text { Hedges' } \boldsymbol{g})\end{array}$ \\
\hline Depression & $9.63(10.04)$ & $6.14(6.92)$ & 1.95 & 3.50 & $-0.14,7.12$ & 0.32 & $-0.14,0.77$ \\
Anxiety & $4.32(5.63)$ & $4.80(4.70)$ & -0.53 & -0.59 & $-2.33,-1.37$ & -0.09 & $-0.54,0.36$ \\
Stress & $14.58(8.97)$ & $10.29(8.16)$ & 2.94 & 4.29 & $1.34,-7.24$ & 0.48 & $0.02,0.93$ \\
\hline
\end{tabular}

Notes.

Norms based on Females' normative data sample $(N=1,870)$, Lovibond \& Lovibond (1995b). Hedges' effect size: small (0.2) medium (0.5) and large (0.8). Tests on the four groups were conducted using the Bonferroni adjustment alpha levels of .016 (.05/3).

${ }^{*} p<.012$, two-tailed.

The data indicated higher levels of stress in the non-SASR members than in the normative data (see Table 4).

Sample 1 reported mean scores in the moderate range for depression $(M=16.09)$, anxiety $(M=12.61)$, and stress $(M=19.69)$, and the Sample 2 depression mean scores $(M=18.84)$, anxiety $(M=12.88)$ and stress $(M=22.68)$ were also in the moderate range. Sample 3 were in the non-clinical range for all categories: depression $(M=4.15)$, anxiety $(M=2.45)$, and stress $(M=9.65)$. Sample 4 reported values in the non-clinical range for depression $(M=9.64)$ and anxiety $(M=5.59)$, but marginally in the mild category for stress $(M=14.31)$. The severity ratings for DASS normative data (Lovibond \& Lovibond, 1995a) are presented in Table 5. 


\begin{tabular}{llll}
\hline Table 5 & DASS 42 severity ratings taken from Lovibond \& Lovibond (1995b). & \\
Rating & Depression & Anxiety & Stress \\
\hline Normal & $0-9$ & $0-7$ & $0-14$ \\
Mild & $10-13$ & $8-9$ & $15-18$ \\
Moderate & $14-20$ & $10-14$ & $19-25$ \\
Severe & $21-27$ & $15-19$ & $26-33$ \\
Extremely severe & $28+$ & $20+$ & $34+$ \\
\hline
\end{tabular}

\begin{tabular}{|c|c|c|c|c|c|c|}
\hline \multirow[t]{2}{*}{ Sample } & \multirow[t]{2}{*}{$n$} & \multirow[t]{2}{*}{$M$} & \multirow[t]{2}{*}{$S D$} & \multicolumn{3}{|c|}{$r$} \\
\hline & & & & Depression & Anxiety & Stress \\
\hline Sample 2: POV & 40 & 2.63 & 1.23 & .16 & .01 & .13 \\
\hline Sample 3: SASR & 40 & 6.40 & 3.89 & .18 & .04 & .22 \\
\hline Sample 4: Non-SASR & 38 & 3.95 & 4.03 & $.35^{*}$ & $.39^{*}$ & $.40^{*}$ \\
\hline
\end{tabular}

Notes.

POV, Partners of veterans; SASR, Special Air Services Regiment

${ }^{*} p<.05$, two-tailed.

As expected, Table 6 shows that Sample 3 reported a higher number of deployments than (a) Sample 2, Hedges' $g=1.33$ (95\% CI [0.87-1.78]) and (b) Sample 4, Hedges' ( $g=0.77$ [95\% CI [0.31-1.22]). However, the correlations between the number of deployments and depression, anxiety, and stress measures were large and statistically significant for Sample 4 only, see Table 6 .

\section{DISCUSSION}

The present study compared three of the major symptoms of psychosocial dysfunction (depression, anxiety and stress) in partners of Australian combat veterans with Australian normative data. It also examined some of the similarities and differences within different samples of those partners. To date, there has been no comparison of mental health symptomatology relative to normative data, nor have there been comparisons between different groups of partners of Australian veterans. The present paper provides a preliminary snapshot of the psychological distress experienced by these groups of partners of veterans. The results did not fully support our prediction that the more deployments a veteran had, the greater psychosocial dysfunction the partner would experience. Number of deployments was not related to depression, anxiety, or stress in the POV or the SASR samples. However, the higher number of deployments was associated with significantly greater levels of depression, anxiety, and stress in the partners of non-SASR veterans. This could indicate that factors related to number of deployments such as constant moving of location and poor social support satisfaction and networks may be the underlying factors affecting depression, anxiety, and stress in these partners. 
Many researchers have found that the health of the partner impacts significantly on the health outcomes of the veterans (Ahmadi \& Green, 2011) and that partners and family units are the principal support systems for veterans (Renshaw, Rodebaugh \& Rodrigues, 2010). However, limited emphasis has been put on comparative analysis of the psychological health of partners relative to Australian normative data. Results indicated that those in the older mean age samples comprising partners of Australian veterans (Samples 1 and 2) reported greater symptomatology as compared with normative data than those in the younger samples comprising partners of currently serving military personnel (Samples 3 and 4). This may be from long-term caring responsibilities and the longer length of time of living with a veteran (Outram et al., 2009), and the results may have implications for support services for the partners and families of combat veterans. For example, preventative programs may be more appropriate for the younger partners, whereas more clinical and respite support services may be required for the older partners.

It should also be noted that partners of SASR members had lower levels of depression and stress, than the Australian normative data, although the effect sizes were small. Partners of SASR members have the ability to stay in the same location compared to the non-SASR partners. Some non-SASR partners can be relocated every two to three years from one side of Australia to another and have to form new relationships and support systems with each move. Constant relocation combined with multiple deployments may lead to higher levels of stress (Australian National Audit-Office, 2012). The majority of the SASR-cohort partners were professionals with a solid career. Constant relocations have shown to have a detrimental effect on partners of non-SASR career and employment opportunities (Australian National Audit-Office, 2012). This could serve as another life stressor for the partners. Our results show that current-serving non-SASR partners in this cohort reported elevated stress levels compared to the Australian normative data. This could reflect a constant state of hyperarousal in the partners, which reflects the issues and problems they experience in meeting their daily life demands (Lovibond \& Lovibond, 1995b.).

\section{Limitations and future research}

First, only a single measure (DASS) was used to examine mental health symptoms. Future research could include other measures to assess mental health problems as well as positive concepts such as psychological and physical well-being. Second, the present study used a convenience sample, which could potentially have biased the results. Third, the sample sizes were relatively small in Samples 2, 3, and 4 and Sample 3 had lower Cronbach's alpha levels for the DASS subscales. Future research could examine several different concepts more carefully such as age, number of relocations, and career. Future research might also like to employ a large cohort of partners and veterans in a longitudinal study, thus examining outcomes such as suicide and prevention not only for veterans (e.g., Yi \& Hong, 2015) but for their partners as well. More research needs to be done on partners of the different armed forces such as Army, Navy, Air Force and Australian Federal Police who serve overseas. 


\section{Conclusion}

Mental health of partners of Australian veterans is generally poorer in terms of greater depression, anxiety and stress levels than the comparative norms. However, partners of SASR personnel seemed to have lower levels of depression and anxiety than the comparative norms. Elevated levels of depression, anxiety, and stress appear to be associated with higher number of deployments in non-SASR partners but this relationship may be explained by other factors as this relationship did not hold in the POV or SASR samples.

\section{ADDITIONAL INFORMATION AND DECLARATIONS}

\section{Funding}

The authors received no funding for this work.

\section{Competing Interests}

The authors declare there are no competing interests.

\section{Author Contributions}

- Gail V. MacDonell, Navjot Bhullar and Einar B. Thorsteinsson conceived and designed the experiments, performed the experiments, analyzed the data, contributed reagents/materials/analysis tools, wrote the paper, prepared figures and/or tables, reviewed drafts of the paper.

\section{Human Ethics}

The following information was supplied relating to ethical approvals (i.e., approving body and any reference numbers):

Human Research Ethics Committee, University of New England, approval number HE09/151.

\section{Data Availability}

The following information was supplied regarding data availability:

MacDonell GV, Bhullar N \& Thorsteinsson EB (2016a). De-identified DASS norms POV282 [DATA]. figshare. Available at: http://dx.doi.org/10.6084/m9.figshare.3120697.

MacDonell GV, Bhullar N, \& Thorsteinsson EB (2016b). De-identified DASS norms three groups (POV50, SASR41, non-SASR38) [DATA]. figshare. Available at: http://dx.doi.org/10.6084/m9.figshare.3120676.

\section{Supplemental Information}

Supplemental information for this article can be found online at http://dx.doi.org/10.7717/ peerj.2373\#supplemental-information.

\section{REFERENCES}

Ahmadi H, Green SL. 2011. Screening, brief intervention, and referral to treatment for military spouses experiencing alcohol and substance use disorders: a literature review. Journal of Clinical Psychology in Medical Settings 18(2):129-136 DOI 10.1007/s10880-011-9234-7. 
Alessi MW, Ray JW, Ray GE, Stewart SJ. 2001. Personality and psychopathology profiles of veterans' wives: measuring distress using the MMPI-2. Journal of Clinical Psychology 57(12):1535-1542 DOI 10.1002/jclp.1115.

Antony MM, Bieling PJ, Cox BJ, Enns MW, Swinson RP. 1998. Psychometric properties of the 42-item and 21-item versions of the depression anxiety stress scales in clinical groups and a community sample. Psychological Assessment 10:176-181.

Australian National Audit-Office. 2012. Delivery of bereavement and family support services through the Defence Community Organisation. (Report No. 9 2012-13). Available at http://www.aph.gov.au/DocumentStore.ashx?id=925f1e16-d838-4c52ab04-f05ab12d762e.

Beckham JC, Lytle B, Feldman M. 1996. Caregiver burden in partners of Vietnam war veterans with posttraumatic stress disorder. Journal of Consulting and Clinical Psychology 64(5):1066-1072 DOI 10.1037/0022-006X.64.5.1068.

Burrell LM, Adams GA, Durand DB, Castro CA. 2006. The impact of military lifestyle demands on well-being, army, and family outcomes. Armed Forces \& Society 33(1):43-58 DOI 10.1177/0002764206288804.

Calhoun PS, Beckham JC, Bosworth HB. 2002. Caregiver burden and psychological distress in partners of veterans with chronic posttraumatic stress disorder. Journal of Traumatic Stress 15(3):205-212 DOI 10.1023/A:1015251210928.

Dekel R, Goldblatt H, Keidar M, Solomon Z, Polliack M. 2005. Being a wife of a veteran with posttraumatic stress disorder. Family Relations 54(1):24-36 DOI 10.1111/j.0197-6664.2005.00003.x.

Dekel R, Solomon Z, Bleich A. 2005. Emotional distress and marital adjustment of caregivers: contribution of level of impairment and appraised burden. Anxiety, Stress \& Coping 18(1):71-82 DOI 10.1080/10615800412336427.

Dirkzwager AJE, Bramsen I, Adèr H, Van der Ploeg HM. 2005. Secondary traumatization in partners and parents of Dutch peacekeeping soldiers. Journal of Family Psychology 19(2):217-226 DOI 10.1037/0893-3200.19.2.217.

Doherty C, Patton W, Shield P. 2015. Family mobility: reconciling career opportunities and educational strategy: changing Mobilities. New York: Routledge.

Frančikovišć T, Stevanovic A, Jelušić I, Roganović B, Klarić M, Grković J. 2007. Secondary traumatization of wives of war veterans with posttraumatic stress disorder. Croatian Medical Journal 48(2):177-184.

Hoops JR. 2012. The effects of multiple combat-related military deployments on post traumatic stress symptoms. Master of Social Work Clinical Research Papers 5:1-47.

Kline A, Falca-Dodson M, Sussner B, Ciccone DS, Chandler H, Callahan L, Losonczy M. 2010. Effects of repeated deployment to Iraq and Afghanistan on the health of New Jersey Army National Guard Troops: implications for military readiness. American Journal of Public Health 100(2):276-283 DOI 10.2105/AJPH.2009.162925.

Lewis M, Lamson A, Leseuer B. 2012. Health dynamics of military and veteran couples: a biopsychorelational overview. Contemporary Family Therapy 34(2):259-276 DOI 10.1007/s10591-012-9193-7. 
Link PE, Palinkas LA. 2013. Long-term trajectories and service needs for military families. Clinical Child and Family Psychology Review 16(4):376-393 DOI 10.1007/s10567-013-0145-z.

Lovibond PF, Lovibond SH. 1995a. The structure of negative emotional states: comparison of the Depression Anxiety Stress Scales (DASS) with the beck depression and anxiety inventories. Behaviour Research and Therapy 33(3):335-343 DOI 10.1016/0005-7967(94)00075-U.

Lovibond SH, Lovibond PF. 1995b. Manual for the depression anxiety stress scales. Sydney, NSW: Psychology Foundation of Australia.

MacDonell GV, Bhullar N, Thorsteinsson EB. 2016a. De-identified DASS norms POV282 [DATA]. Figshare DOI 10.6084/m9.figshare.3120697.

MacDonell GV, Bhullar N, Thorsteinsson EB. 2016b. De-identified DASS norms three groups (POV50, SASR41, non-SASR38) [DATA]. Figshare DOI 10.6084/m9.figshare.3120676.

MacDonell G, Marsh NV, Hine DW, Bhullar N. 2010. The development and psychometric evaluation of a measure to assess distress in partners of Australian combat veterans. Australian and New Zealand Journal of Psychiatry 44(9):839-845 DOI 10.3109/00048674.2010.488214.

MacDonell G, Thorsteinsson EB, Bhullar N, Hine DW. 2014. Psychological functioning of partners of Australian Combat veterans: contribution of veterans' PTSD symptoms and partners' caregiving distress. Australian Psychologist 49:305-312 DOI 10.1111/ap.12069.

Outram S, Hansen V, MacDonell G, Cockburn J, Adams J. 2009. Still living in a war zone: perceived health and wellbeing of partners of Vietnam veterans attending partners' support groups in NSW, Australia. Australian Psychologist 44(2):128-135 DOI 10.1080/00050060802630353.

Padden DL, Connors RA, Agazio JG. 2011. Stress, coping, and well-being in military spouses during deployment separation. Western Journal of Nursing Research 33(2):247-267 DOI 10.1177/0193945910371319.

Pearlin L, Mullan J, Semple S, Skaff M. 1990. Caregiving and the stress process: an overview of concepts and their measures. The Gerontologist 30(5):583-594 DOI 10.1093/geront/30.5.583.

Renshaw KD, Campbell SB. 2011. Combat veterans' symptoms of PTSD and partners' distress: the role of partners' perceptions of veterans' deployment experiences. Journal of Family Psychology 25(6):953-962 DOI 10.1037/a0025871.

Renshaw KD, Caska CM. 2012. Relationship distress in partners of combat veterans: the role of partners' perceptions of posttraumatic stress symptoms. Behavior Therapy 43(2):416-426 DOI 10.1016/j.beth.2011.09.002.

Renshaw KD, Rodebaugh TL, Rodrigues CS. 2010. Psychological and marital distress in spouses of Vietnam veterans: importance of spouses' perceptions. Journal of Anxiety Disorders 24(7):743-750 DOI 10.1016/j.janxdis.2010.05.007. 


\section{Salimi SH, Azad-Marazbadi E, Karaminia R, Mirzamani SM, Hosseini-Sangtrashani}

S. 2006. Being a wife of a veteran with psychiatric problem or chemical warfare exposure: a preliminary report from Iran. Iranian Journal Psychiatry 1(2):65-69.

Solomon Z, Greene T, Ein-Dor T, Zerach G, Benyaminini Y, Ohry A. 2014. The longterm implications of war captivity for mortality and health. Journal of Behavioral Medicine 37(5):849-859 DOI 10.1007/s10865-013-9544-3.

Solomon Z, Mikulincer M. 2006. Trajectories of PTSD: a 20-year longitudinal study. The American Journal of Psychiatry 163(4):659-666 DOI 10.1176/ajp.2006.163.4.659.

Solomon Z, Waysman M, Avitzur E, Enoch D. 1991. Psychiatric symptomology among wives of soldiers following combat stress reaction: the role of the social network and marital relations. Anxiety Research 4(3):213-223 DOI 10.1080/08917779108248775.

Tabachnick BG, Fidell LS. 2001. Using multivariate statistics. Sydney: Allyn and Bacon.

Westerink J, Giarratano L. 1999. The impact of posttraumatic stress disorder on partners and children of Australian Vietnam veterans. Australian and New Zealand Journal of Psychiatry 33:841-847 DOI 10.1046/j.1440-1614.1999.00638.x.

Yi S, Hong J. 2015. Depressive symptoms and other risk factors predicting suicide in middle-aged men: a prospective cohort study among Korean Vietnam War veterans. PeerJ 3:e1071 DOI 10.7717/peerj.1071. 